\title{
Note on anomalies in the AdS-CFT correspondence
}

\author{
Ofer Aharony \\ Department of Physics and Astronomy, Rutgers University, Piscataway, New Jersey 08855 \\ Jacek Pawełczyk* and Stefan Theisen \\ Institut für Theoretische Physik, Universität München, Theresienstraße 37, 80333 München, Germany \\ Shimon Yankielowicz \\ School of Physics and Astronomy, Beverly and Raymond Sackler Faculty of Exact Sciences, Tel-Aviv University, Ramat Aviv, \\ Tel-Aviv 69978, Israel \\ (Received 3 February 1999; published 2 August 1999)
}

\begin{abstract}
We test the AdS-CFT correspondence in the case of a $d=4 \mathcal{N}=2$ superconformal field theory by comparing chiral anomalies which are of order $N$ in the 't Hooft large $N$ limit. These include corrections of order $1 / N$ to the conformal anomaly, thus testing the correspondence beyond the extreme large $N$ limit. The field theory anomalies are reproduced by terms in the 7-brane effective action in the bulk. [S0556-2821(99)05514-9]
\end{abstract}

PACS number(s): 11.25.Hf, 11.15.Pg

\section{INTRODUCTION AND SUMMARY}

Over the past year much evidence has accumulated for the conjecture of [1] relating $d$-dimensional conformal field theories with compactifications of string theory or M theory including $\mathrm{AdS}_{d+1}$. The simplest example of the correspondence is the duality between the $d=4 \mathcal{N}=4 S U(N)$ Super Yang-Mills (SYM) theory and type IIB string theory compactified on $\mathrm{AdS}_{5} \times \mathrm{S}^{5}$. This duality relates the $S U(N)$ gauge theory with coupling $\tau_{Y M} \equiv \theta / 2 \pi+4 \pi i / g_{Y M}^{2}$ to the type IIB compactification with a string coupling $\tau_{s} \equiv \chi / 2 \pi+i / g_{s}$ $=\tau_{Y M}$ and with $N$ units of 5-form flux on the $S^{5}$, leading to a radius of curvature $R \sim\left(g_{s} N\right){ }^{1 / 4} l_{s} \sim N^{1 / 4} l_{p}$. The correlation functions of local operators in the conformal field theory are related $[2,3]$ to the response of string theory on AdS to various boundary conditions.

In field theory, it is well known that correlation functions have a $1 / N$ expansion based on the double-line representation of their Feynman diagrams [4] (see [5] for a review). This expansion is valid in the limit of taking large $N$ and small $g_{Y M}$ while keeping $\lambda \equiv g_{Y M}^{2} N$ finite. A diagram with genus $g$ comes with a power $N^{2-2 g}$ as well as some power of $\lambda$. For $S U(N)$ theories with adjoint fields only closed orientable surfaces appear, so the perturbative expansion is a double expansion in powers of $1 / N^{2}$ and $\lambda$, in which the leading term (corresponding to the planar diagrams) is of order $N^{2}$

In the dual AdS theory we find a similar result in the 't Hooft limit of large $N$ with finite $\lambda \sim g_{s} N$. In general, correlation functions in the $\operatorname{AdS}_{5} \times \mathrm{S}^{5}$ string theory are given by a double expansion in $g_{s}$ and $\alpha^{\prime} / R^{2} \sim \lambda^{-1 / 2}$. In the 't Hooft limit we can write this instead as an expansion in powers of $1 / N \sim g_{s}\left(\alpha^{\prime} / R^{2}\right)^{2}$ where each coefficient has an expansion in powers of $\lambda^{-1 / 2}$; obviously a term of some order in the $g_{s}$

\footnotetext{
* On leave from the Institute of Theoretical Physics, Warsaw University.
}

expansion will have the same order in the $1 / N$ expansion. Since the type IIB string theory includes only closed oriented strings we find the same general structure as in the field theory, with each correlation function having an expansion in powers of $1 / N^{2}$, in which each coefficient is some function of $\lambda$.

The functions of $\lambda$ have different expansions from the point of view of the field theory and of the string theory, but both expansions are supposed [if the AdS-conformal field theory (CFT) correspondence is correct] to give rise to the same function of $\lambda$ at each order in $1 / N^{2}$. The fact that one expansion is in powers of $\lambda$ while the other is in powers of $1 / \sqrt{\lambda}$ means that the AdS-CFT correspondence is a strongweak coupling duality in this case. Since we do not know how to make computations to arbitrary order in $\lambda$, this means that only correlation functions which do not depend on $\lambda$ can be compared to test the duality. Many such tests have been done by now for terms of order $N^{2}$, which on the AdS side may be computed from tree-level supergravity. However, it is important to test this matching also at higher orders in $1 / N$ in order to make sure that the duality of [1] indeed holds also for finite $N$ and not just in the large $N$ limit (some evidence for this was provided in $[6,7])$. As far as we know, the only computations performed up to now of higher order terms (in $1 / N$ ) have involved instanton corrections $[8,9,10,11]$ (some other corrections were recently discussed but not explicitly computed in [12]).

The simplest correlation functions which do not depend on $\lambda$ are those which correspond to global anomalies. Namely, when we put the theory in backgrounds corresponding to curved space or to gauge fields coupling to the global currents, some of the global currents are no longer conserved due to anomalies, and the coefficients in the expressions for this are integers so they cannot depend on $\lambda$. In the $\mathcal{N}=4$ theory there are two such anomalies, which were both successfully matched to leading order in $1 / N^{2}$. The anomaly involving three $S U(4)_{R}$ currents was discussed in $[3,13]$, and the conformal anomaly was discussed in [14]. In the $S U(N)$ SYM theory both anomalies are proportional to 
$N^{2}-1$, and the order $N^{2}$ term has been matched, while it is not known how to derive the order 1 term on the AdS side (because of our lack of control over the string loop corrections in this case; note that in general it is easy to obtain the exact expressions for the anomalies on the field theory side, but it is nontrivial to get them on the string theory-M theory side). Since the comparison of the terms of order $1 / N^{2}$ compared to the leading term seems to be difficult, we would like to examine situations where there exist also corrections of order $1 / N$. In this paper we compute some corrections of this order and show that they agree between the field theory and the string theory, providing further evidence for the AdSCFT correspondence at finite $N$.

From the string theory point of view it is clear that in order to get diagrams of order $1 / N$ one must have either open string diagrams or nonorientable diagrams. This is wellknown also in the field theory analysis of the 't Hooft limit, where looking at $S O(N)$ or $U S p(2 N)$ gauge theories leads to nonorientable diagrams with contributions of order $1 / \mathrm{N}$, while adding matter in the fundamental representation (but not in bifundamental representations) leads to diagrams with boundaries which also have contributions of order $1 / N$. On the AdS side such corrections can occur due to orientifolds which lead to the inclusion of nonorientable worldsheets, or due to D-branes which lead to worldsheets with boundaries.

Note that in all cases of orbifolds and orientifolds the comparison of the anomalies in the leading $N^{2}$ order is straightforward. For example, it was shown in [15] that the leading term in the conformal anomaly on the AdS side is inversely proportional to the volume of the compact space, so that for a $\mathbf{Z}_{k}$ orientifold or orbifold the leading term is $k$ times the $\mathcal{N}=4$ result. This obviously agrees with the field theory analysis of D3-branes on codimension $6 \mathbf{Z}_{2}$ orientifolds [16] which lead to $S O(2 N), S O(2 N-1)$ or $U S p(2 N)$ gauge theories (with a leading anomaly of order $2 N^{2}$ ), and with the analysis of D3 branes on codimension $4 \mathbf{Z}_{k}$ orbifolds [17] which correspond to $S U(N)^{k}$ gauge theories (with a leading anomaly of order $k N^{2}$ ).

In particular, the analysis of $[14,15]$ shows that the supergravity computation always leads to the two coefficients appearing in the conformal anomaly (usually denoted by $a$ and c) being equal to each other, so that duals with a useful supergravity limit can exist only for theories for which $a$ $=c$ to leading order in $1 / N$. We will show that when higher order corrections are taken into account this no longer has to hold, so the constraint $a=c$ is only required at the leading order in $1 / N$ (of course, there could also be cases of the duality which have no good supergravity approximation, in which case there is no obvious relation between $a$ and $c$ ).

The simplest case where an order $1 / N$ correction to anomalies exists is the near-horizon limit of D3-branes on an orientifold 3-plane analyzed in [16]. However, in this case the correction on the string theory side comes from an $R P^{2}$ diagram which, to our knowledge, has not been computed yet. Therefore, we will focus here on the next simplest case, which is the near-horizon limit of D3-branes on a $\mathbf{Z}_{2}$ orientifold 7-plane (with 8 D7-branes stuck on the orientifold to ensure conformal invariance). The $\mathcal{N}=2$ superconformal field theory corresponding to this case was analyzed in $[18,19,20,21]$ and its string theory dual was analyzed in $[22,23]$. We will see that in this case we can compute some of the order $1 / N$ corrections on the string theory side as well as on the field theory side, by using the effective D7-brane action [whose leading terms are of order $1 / g_{s} \propto N$ instead of the $1 / g_{s}^{2} \propto N^{2}$ appearing in the supergravity (SUGRA) action], and we will show that the string theory and field theory results agree to this order. We have not been able to compute all of the anomalies to this order, and in particular we do not know how to directly reproduce the conformal anomaly, but it is related by supersymmetry to the anomaly terms that we do compute, so supersymmetry guarantees that the conformal anomaly also agrees. Our results may presumably be generalized also to the other cases involving D7-branes and orientifolds which were discussed in [22,23], for which it is not known how to compute the anomalies on the field theory side, so the string theory computation is a prediction of what these anomalies should be.

In Sec. II we describe the model and the anomalies of its $U$ (1) $R$-current from the field theory point of view. In Sec. III we analyze how these anomalies are related to ChernSimons (CS) terms in the effective action of the D7-branes. The results of Secs. II and III are compared in Sec. IV. This requires a careful fixing of the normalizations, which involves a comparison of the two-point correlation functions of the $R$-current and the flavor current as computed in the field theory and as computed via the AdS-CFT correspondence. Unless stated otherwise, we will use the conventions of [24].

\section{ANOMALIES IN THE $\mathcal{N}=2$ SUPERCONFORMAL FIELD THEORY}

The model we are considering is the one constructed in $[20,21]$, namely the low-energy theory on the world volume of $N$ D3-branes sitting inside eight D7-branes coincident on an orientifold 7-plane. This theory is dual $[22,23]$ to type IIB string theory on $\operatorname{AdS}_{5} \times X^{5}$ where $X^{5} \simeq S^{5} / Z_{2}$; the local operators in the field theory can be thought of as living on $\operatorname{Mink}(3,1)=\partial\left(\mathrm{AdS}_{5}\right)$. The D7-branes (and the orientifold 7-plane) are wrapped around an $S^{3}$ which is the fixed point locus of the $\mathbf{Z}_{2}$ orientifold inside $\mathrm{X}^{5}$, and also fill the whole of $\mathrm{AdS}_{5}$. The low-energy theory in the bulk of $\mathrm{AdS}_{5}$ includes the gauged $S U(2)_{R} \times U_{\mathcal{R}}(1) \quad \mathcal{N}=4$ supergravity, coupled to $S O(8) \times S U(2)_{L}$ vector multiplets. Further details about these models and their dual string theory description are in $[22,23]$. Some aspects relevant for our discussion will be reviewed below. The $U_{\mathcal{R}}(1)$ symmetry whose anomalies we study is the one in the inclusion

$$
S O(6) \supset S O(4) \times U_{\mathcal{R}}(1) \simeq S U(2)_{R} \times S U(2)_{L} \times U_{\mathcal{R}}(1)
$$

of the isometry group of $S^{5} / Z_{2}$ in the isometry group of $S^{5}$.

The field theory we are analyzing is a $d=4, \mathcal{N}=2$ gauge theory with a $U S p(2 N)$ gauge group and with the following field content:

$$
\begin{array}{ll}
\text { vector multiplets } & \mathbf{N}(\mathbf{2} \mathbf{N}+\mathbf{1}) \quad \text { (adjoint), } \\
\text { hypermultiplets } & 4 \times \mathbf{2} \mathbf{N} \quad \text { (fundamental), }
\end{array}
$$


$\mathbf{N}(\mathbf{2 N}-\mathbf{1})-\mathbf{1}$ (antisymmetric, traceless).

Its global symmetry is $S U(2)_{R} \times S U(2)_{L} \times S O(8)$ $\times U_{\mathcal{R}}(1)$, where $S U(2)_{R} \times U_{\mathcal{R}}(1)$ is the $R$-symmetry (which is part of the $\mathcal{N}=2$ superconformal algebra). Here we concentrate on anomalies of the $U_{\mathcal{R}}(1) R$-current $\mathcal{R}^{\mu}$. The form of the unique anomaly-free $\mathcal{R}^{\mu}$ current is specified by the $\mathcal{R}$-charges for the vector multiplet fermions $\lambda_{i}, i=1,2(Q$ $=+1)$, the bosons $\phi(Q=+2)$ in the vector multiplets, and the matter (hypermultiplet) fermions $\psi, \widetilde{\psi}(Q=-1)$ :

$$
\mathcal{R}_{\mu}=\frac{1}{2} \bar{\lambda}_{i} \gamma_{\mu} \gamma_{5} \lambda_{i}-\frac{1}{2}\left(\bar{\psi} \gamma_{\mu} \gamma_{5} \psi+\widetilde{\bar{\psi}} \gamma_{\mu} \gamma_{5} \widetilde{\psi}\right)-2 i \bar{\phi} \stackrel{\leftrightarrow}{D}_{\mu} \phi
$$

The factor $1 / 2$ in front of the fermion bilinears is due to the fact that $\lambda_{i}, \psi$ and $\widetilde{\psi}$ are Majorana spinors.

The current (2.3) is anomalous when the theory is coupled to gravity. The anomaly can be calculated using the general result of [25]: a single Weyl fermion with $U(1)$ charge $Q$ contributes to the anomaly as

$$
\left\langle\partial_{\mu}\left(\sqrt{g} \mathcal{R}^{\mu}\right)\right\rangle=\frac{Q}{384 \pi^{2}}(\widetilde{R} R),
$$

where

$$
(\widetilde{R} R)=\frac{1}{2} \epsilon_{\mu \nu \rho \sigma} R_{\delta \gamma}^{\mu \nu} R^{\rho \sigma \delta \gamma} .
$$

For the model we are considering, we find

$$
\left\langle\partial_{\mu}\left(\sqrt{g} \mathcal{R}^{\mu}\right)\right\rangle=\frac{2(1-6 N)}{384 \pi^{2}}(\widetilde{R} R) .
$$

For $\mathcal{N}=2$ SUSY theories the one-loop result (2.6) is not renormalized. ${ }^{1}$ Supersymmetry relates the chiral anomaly $\left\langle\partial_{\mu}\left(\sqrt{g} \mathcal{R}^{\mu}\right)\right\rangle$ and the conformal anomaly $\left\langle g^{\mu \nu} T_{\mu \nu}\right\rangle$ (see, for instance, $[26,27,28,29])$. They are both in the same supersymmetry multiplet, and therefore the conformal anomaly is also exact at one loop in this theory. The coefficient on the right hand side of Eq. (2.6) is proportional to $a-c=(1$ $-6 N) / 24$, where $\left\langle g^{\mu \nu} T_{\mu \nu}\right\rangle=-a E_{4}-c I_{4}$, and $E_{4}$ and $I_{4}$ are (proportional to) the Euler density and the square of the Weyl tensor, respectively (see, e.g., $[14,15]$ ). Note that $a$ and $c$ are both of order $N^{2}$ in the large $N$ limit (as computed for the $\mathcal{N}=4$ case in [14], a similar computation in our case gives $a=c=2 N^{2} / 4$ in the large $N$ limit). Therefore, the coefficient computed above involves corrections of order $1 / N$ compared to the leading term in $a$ and $c$.

In addition to the above gravitational contribution to the anomalous divergence of the $U(1)_{\mathcal{R}}$ current, there is also a contribution from the $\langle\mathcal{R} J J\rangle$ triangle diagram, where $J$ is the

\footnotetext{
${ }^{1}$ Note that, in contrast with generic $\mathcal{N}=1$ theories, the $R$-current which is in the supermultiplet of currents is also the one which satisfies the Adler-Bardeen theorem.
}

$S O(8)$ flavor current, if we couple the theory to external $S O(8)$ gauge fields. Since only the "quark" hypermultiplets are charged under $S O(8)$, this gives

$$
\left\langle\partial_{\mu}\left(\sqrt{g} \mathcal{R}^{\mu}\right)\right\rangle=\frac{2 N}{16 \pi^{2}}(\widetilde{F} F),
$$

where $(\widetilde{F} F)=1 / 2 \epsilon_{\mu \nu \rho \sigma} \operatorname{tr}\left(F^{\mu \nu} F^{\rho \sigma}\right), F=F^{a} t^{a}$, and the trace is taken in the fundamental representation. We will fix the normalization of the $S O(8)$ generators later by comparing the field theory two-point function of the $S O(8)$ flavor current with the two-point function computed from string theory using the AdS-CFT correspondence. Combining the two contributions (2.6) and (2.7) gives, to leading order in $1 / N$,

$$
\left\langle\partial_{\mu}\left(\sqrt{g} \mathcal{R}^{\mu}\right)\right\rangle=-\frac{N}{32 \pi^{2}}[(\widetilde{R} R)-4(\widetilde{F} F)] .
$$

\section{ANOMALIES FROM FIVE-DIMENSIONAL CHERN-SIMONS TERMS}

We will now show how the $R$-current anomalies can be obtained from the string theory dual of this theory. Our procedure will be completely analogous to the one used in [3] - the anomalies will be related to Chern-Simons (CS) terms in the five-dimensional effective action. Chern-Simons terms can arise both from the dimensionally reduced $d=10$ type IIB supergravity and from the 7-brane-orientifold plane system. The former gives possible anomalous contributions to $\left\langle\mathcal{R}^{3}\right\rangle$ and $\left\langle\mathcal{R} \mathcal{J}^{2}\right\rangle$, where $\mathcal{J} \mathrm{s}$ are the $S U(2)$ currents. They are of order $N^{2}$ in the large $N$ expansion (since the whole $d=10$ supergravity action is of this order). We will not consider them here. The second source for CS terms are the D7-branes and the orientifold plane, which are both wrapped around an $S^{3}$. As we shall see these terms are of order $N$.

We now focus on the five-dimensional terms which arise from dimensional reduction of the Chern-Simons terms, which appear in the world-volume action of the 7-branes, on the internal $S^{3}$. The CS terms for a general $D p$-brane are [30]

$$
\left.\mu_{p} \int C \wedge \sqrt{\hat{A}\left(4 \pi^{2} \alpha^{\prime} R\right)} \operatorname{tr}\left(e^{2 \pi \alpha^{\prime} F}\right)\right|_{p+1},
$$

where $\mu_{p}$ is the charge of a single $D p$-brane, $C=\Sigma C_{(n)}$ is the sum over the antisymmetric form $R R$ fields present in the theory and ${ }^{2} F=F^{a} T^{a}$ is the field strength of the gauge fields on the $D p$-brane. In our case these are the $S O(8)$ gauge fields. The trace is in the fundamental representation. The orientifold plane also gives rise to CS terms on the brane world volume. They have recently been determined to be $[31,32]$

$$
\left.\mu_{p}^{\prime} \int C \wedge \sqrt{\hat{L}\left(\pi^{2} \alpha^{\prime} R\right)}\right|_{p+1}
$$

\footnotetext{
${ }^{2}$ Note that we distinguish between $t^{a}$ in Eq. (2.7) and $T^{a}$ here. Their relative normalization will be fixed in Sec. IV.
} 
where $\mu_{p}^{\prime}$ is the charge of an orientifold $p$-plane. We have suppressed possible contributions from the Neveu-SchwarzNeveu-Schwarz (NS-NS) $B$ field and also the contribution from the normal bundle, which are of no relevance for our considerations. Up to the required order,

$$
\begin{aligned}
& \hat{A}(R)=1+\frac{1}{(4 \pi)^{2}} \frac{1}{12} \operatorname{tr}(R \wedge R), \\
& \hat{L}(R)=1-\frac{1}{(2 \pi)^{2}} \frac{1}{6} \operatorname{tr}(R \wedge R) .
\end{aligned}
$$

First, we would like to determine the coefficients $\mu_{p}$ and $\mu_{p}^{\prime}$. Before we take the near-horizon limit, the model we are considering is $T$ dual, say along the $x^{8}, x^{9}$ directions, to the type I string [18], which has one space-filling orientifold plane and 32 nine-branes. After $T$-duality we have four orientifold 7-planes and 32 seven-branes. This model is equivalent to type IIB on $\operatorname{Mink}(7,1) \times T^{2} /\left(I_{89} \cdot(-1)^{F_{L}} \cdot \Omega\right)$ where $\Omega$ is the world-sheet parity and $I_{89}:\left(x^{8}, x^{9}\right) \rightarrow\left(-x^{8},-x^{9}\right)$ (see [18] for details), which in turn is $F$ theory on a particular $K 3$. $F$ theory [33] has 24 7-branes. In our setting four pairs of mutually non-local seven-branes combine to four orientifold planes. The remaining $16 \mathrm{D} 7$-branes are equivalent to the 32 seven-branes in the type I description. The sevenbranes, which are $T$-duals of the type I nine-branes, thus each have half the charge of a type IIB D7-brane, i.e. $\mu_{p}^{I}$ $=1 /\left[2(2 \pi)^{p}\left(\alpha^{\prime}\right)^{(p+1) / 2}\right]$. This can also be shown by carefully tracing the brane tension through $T$-duality: due to the fact that in the type I theory there are only unoriented strings, one has (see, e.g., [34]) $\mu_{9}^{I}=(1 / \sqrt{2}) \mu_{9}^{I I}$. T-dualizing on $T^{2}$ with radii $R$ gives, by comparison of the brane energy per unit non-compact volume, $e^{-\phi} \mu_{9}^{I}(2 \pi R)^{2}=e^{-\phi^{\prime}} \mu_{7}^{I}$. The $T$-dualized dilation $\phi^{\prime}$ is obtained by requiring invariance of Newton's constant, i.e., $\left(e^{-2 \phi^{\prime}} / \alpha^{\prime 4}\right) \frac{1}{2}\left(2 \pi R^{\prime}\right)^{2}$ $=\left(e^{-2 \phi} / \alpha^{\prime 4}\right)(2 \pi R)^{2}$ (the additional factor of $\frac{1}{2}$ is explained in [24], p. 150). With $R^{\prime}=\alpha^{\prime} / R$ one finds $e^{\phi^{\prime}}$ $=(1 / \sqrt{2})\left(\alpha^{\prime} / R^{2}\right) e^{\phi}$. This leads again to $\mu_{7}^{I}=\frac{1}{2} \mu_{7}^{I I}$. Charge neutrality of the type I theory requires $\mu_{p}^{\prime}=-2^{p-4} \mu_{p}$.

Thus, with eight D7-branes ${ }^{3}$ and one orientifold 7-plane one obtains from Eqs. (3.1) and (3.2) the terms

$\frac{1}{512 \pi^{5} \alpha^{\prime 2}} \int C_{(4)} \wedge \operatorname{tr}(R \wedge R)+\frac{1}{128 \pi^{5} \alpha^{\prime 2}} \int C_{(4)} \wedge \operatorname{tr}(F \wedge F)$.

The orientifold 7-plane contributes to the second term only insofar as it leads to an orthogonal rather than a unitary gauge group on the D7-branes.

Next, we need to rescale the 4-form field to agree with the conventions used in the AdS literature (for instance in [35] which we will use in Sec. IV). The ten-dimensional supergravity low-energy effective bulk action is

\footnotetext{
${ }^{3}$ The multiplicity comes from the trace of the zeroth order term in the expansion of the exponent, which is $\operatorname{tr}\left(e^{2 \pi \alpha^{\prime} F}\right)=8$ $+\frac{1}{2}\left(2 \pi \alpha^{\prime}\right)^{2} \operatorname{tr}\left(F^{2}\right)+\cdots$.
}

$$
S=\frac{1}{2 \kappa_{10}^{2}} \int d^{10} x \sqrt{-g}\left(R-\frac{1}{4 \times 5 !}\left(g_{s} F_{(5)}\right)^{2}\right),
$$

where $2 \kappa_{10}^{2}=(2 \pi)^{7} g_{s}^{2} \alpha^{\prime 4}$. We have only kept the fields which are relevant for our discussion. Self-duality of $F_{(5)}$ has to be imposed as an additional constraint. The tendimensional Einstein equation resulting from this action, after imposing self-duality of $F_{5}$ (which, in particular, implies $\left.F_{(5)}^{2}=0\right)$, is $R_{M N}=[1 /(16 \times 3 !)]\left(F_{(5)}^{2}\right)_{M N}$. The solution we are interested in involves the ten-dimensional metric and the self-dual five-form field strength. Due to the presence of the seven-branes, the solution is not $\mathrm{AdS}_{5} \times \mathrm{S}^{5}$ but rather $\mathrm{AdS}_{5}$ $\times X^{5}$, which is dual to the field theory described above. The metric is

$$
d s^{2}=\alpha^{\prime} \sqrt{8 \pi g_{s} N}\left(\frac{d u^{2}}{u^{2}}+u^{2} d x_{\|}^{2}+d \Omega_{5}^{2}\right),
$$

where $d \Omega_{5}^{2}$ is the metric on $X^{5} \simeq S^{5} / Z_{2}$. The five-form field is $F_{\text {abcde }}=32 \pi \alpha^{\prime 2} N \epsilon_{\text {abcde }}$ and $F_{\text {mnpqr }}=32 \pi \alpha^{\prime 2} N \epsilon_{\text {mnpqr }}$, with components along the $\mathrm{X}^{5}$ and the $\mathrm{AdS}_{5}$ directions, respectively. $\epsilon_{a b c d e}$ and $\epsilon_{m n p q r}$ are the volume forms of $\mathrm{X}^{5}$ and $\mathrm{AdS}_{5}$, rescaled to radius one. Note the replacement $N \rightarrow 2 N$ as compared to the $\mathrm{AdS}_{5} \times \mathrm{S}^{5}$ solution. This is due to the fact that we require $(1 / \sqrt{2} \kappa) \int_{X^{5}} F_{(5)}=\sqrt{2 \pi} N$ rather than $(1 / \sqrt{2} \kappa) \int_{S^{5}} F_{(5)}=\sqrt{2 \pi} N$, and $\operatorname{Vol}\left(\mathrm{X}^{5}\right)=\frac{1}{2} \operatorname{Vol}\left(\mathrm{S}^{5}\right)$. To match with the normalizations used in Sec. IV, where we use supergravity results which were derived in a normalization where the overall radius of the space is one [rather than $\left.\alpha^{\prime 1 / 2}\left(8 \pi g_{s} N\right)^{1 / 4}\right]$, and where $F_{(5)}$ is scaled such that the Einstein equations take the form $R_{M N}=\frac{1}{6}\left(F_{(5)}^{2}\right)_{M N}$, we rescale the four-form potential $C_{(4)} \rightarrow 32 \pi \alpha^{\prime 2} N C_{(4)}$. After this rescaling, Eq. (3.4) becomes

$$
\frac{N}{16 \pi^{4}} \int C_{(4)} \wedge \operatorname{tr}(R \wedge R)+\frac{N}{4 \pi^{4}} \int C_{(4)} \wedge \operatorname{tr}(F \wedge F) .
$$

To get the CS terms of the $d=5$ theory, we have to integrate this expression over $S^{3}$, the fixed locus of the $\mathbf{Z}_{2}$ action, which the 7-branes are wrapped around. Let us recall from [36] that the $U(1)_{\mathcal{R}}$ gauge boson $A_{m}^{\mathcal{R}}$ is a linear combination of two Kaluza-Klein modes $B_{m}$ and $\phi_{m}$, one coming from the metric components $g_{m a}$ and the other from the $C_{(4)}$ field:

$$
\begin{aligned}
g_{m a} & \equiv B_{m}(x) Y_{a}(y), \\
C_{m a b c} & \equiv \phi_{m}(x) \epsilon_{a b c}{ }^{d e} D_{d} Y_{e}(y) .
\end{aligned}
$$

Here $x$ and $y$ are coordinates on $\mathrm{AdS}_{5}$ and on $\mathrm{X}^{5}$, respectively, and $Y_{a}(y)$ is a vector spherical harmonic on $\mathrm{X}^{5}$ which we now construct.

The metric on $\mathrm{X}^{5}$ is [23]

$$
d \Omega_{5}^{2}=d \theta^{2}+\sin ^{2} \theta d \phi^{2}+\cos ^{2} \theta d \Omega_{3}^{2},
$$


with $0 \leqslant \theta \leqslant \pi / 2, \phi$ has period $\pi$ due to the orientifolding, and $d \Omega_{3}^{2}$ is the metric on $S^{3}$. The seven-branes are located at $\theta=0$. The $k=1$ vector spherical harmonics on $S^{5}$ are

$$
Y_{a}^{[i j]}=x^{[i} \partial_{a} x^{j]},
$$

where $\left(x^{1}\right)^{2}+\cdots+\left(x^{6}\right)^{2}=1$ and $a=1, \ldots, 5$ labels the coordinates on $S^{5}$. They satisfy $\square Y_{a}=-4 Y_{a}$ with $=g^{m n} \nabla_{m} \nabla_{n}$. The relevant harmonic on $X^{5}$ for the $U(1)_{\mathcal{R}}$ gauge field is $Y_{a}^{[56]} \equiv Y_{a}^{U(1)}\left(\mathbf{Z}_{2}\right.$ acts as $\left.x^{5,6} \rightarrow-x^{5,6}\right)$. In polar coordinates it is $\vec{Y}^{U(1)}=\frac{1}{2} \sin ^{2} \theta \hat{\phi}$. This leads to $C_{m a b c}$ $=\phi_{m}(x) \cos ^{4} \theta \omega_{a b c}$, where $\omega_{a b c}$ is the volume form on the unit three-sphere. Note (for later use) that $\int_{X^{5}} \sqrt{g} g^{a b} Y_{a} Y_{b} d^{5} y=\pi^{3} / 24$.

From [36] it follows that the combination $A_{m} \equiv B_{m}$ $-16 \phi_{m}$ is a massless vector field, whereas $V_{m} \equiv B_{m}+8 \phi_{m}$ is a massive field. We will set $V_{m}$ to zero in the following. Up to a normalization, which we will fix in Sec. IV, $A_{m}$ is the $U(1)_{\mathcal{R}}$ gauge field. Denoting by $A_{m}^{\mathcal{R}}$ the $U(1)_{\mathcal{R}}$ field which couples canonically to the current $\mathcal{R}^{\mu}$ of the previous section, we thus identify $\phi_{m}=-\frac{1}{24} A_{m} \equiv \eta A_{m}^{\mathcal{R}}$ for some constant $\eta$. Then, at $\theta=0$,

$$
C_{m a b c}=\eta A_{m}^{\mathcal{R}} \omega_{a b c},
$$

up to some irrelevant additive terms proportional to $V_{m}$. Integrating Eq. (3.7) over $\mathrm{S}^{3}$ we obtain the $d=5 \mathrm{CS}$ terms

$$
\eta \frac{N}{8 \pi^{2}} \int_{A d S_{5}}\left[A^{\mathcal{R}} \wedge \operatorname{tr}(R \wedge R)+4 A^{\mathcal{R}} \wedge \operatorname{tr}(F \wedge F)\right] .
$$

Under the $U(1)_{\mathcal{R}}$ gauge transformation $A^{\mathcal{R}} \rightarrow A^{\mathcal{R}}+d \Lambda$, this CS term transforms as

$$
-\eta \frac{N}{8 \pi^{2}} \int_{\operatorname{Mink}(3,1)} \Lambda[\operatorname{tr}(R \wedge R)+4 \operatorname{tr}(F \wedge F)],
$$

and this can be related to the field theory anomaly $\left\langle\partial_{\mu}\left(\sqrt{g} \mathcal{R}^{\mu}\right)\right\rangle$ as in $[3,13]$. Note the normalizations $\int \operatorname{tr}(R \wedge R)=-\frac{1}{2} \int(\widetilde{R} R) \sqrt{-g} d^{4} x$ and $\int \operatorname{tr}(F \wedge F)$ $=\frac{1}{2} \int(F \widetilde{F}) \sqrt{-g} d^{4} x$.

In the last section we determine $\eta$ by carefully normalizing the various fields, enabling us to compare the expressions for the anomalies which we obtained from the fourdimensional field theory (2.8) and the dual string theory (3.13).

\section{FIXING THE NORMALIZATIONS}

We start by verifying that the relative coefficients of the background gauge and gravitational contributions to the chiral anomaly coincide in the two computations. Since the numerical relative coefficients are the same, this reduces to showing that the generators in the fundamental of $S O(8)$ which appear in Eqs. (2.8) and (3.13) are normalized in the same way. To this end we first compute the two-point function of the $S O(8)$ flavor current in the field theory, which is

$$
J_{\mu}^{a}(x)=\sum_{i=1}^{2 N}\left(-\frac{1}{2} \bar{\psi}_{i} \gamma_{\mu}\left(1-\gamma_{5}\right) t^{a} \psi_{i}+\bar{\phi}_{i} \stackrel{\leftrightarrow}{D}_{\mu} t^{a} \phi_{i}\right)
$$

One finds at one loop

$$
\left\langle J_{\mu}^{a}(x) J_{\nu}^{b}(0)\right\rangle=2 N \operatorname{tr}\left(t^{a} t^{b}\right) \frac{1}{(2 \pi)^{4}}\left(\eta_{\mu \nu} \square-\partial_{\mu} \partial_{\nu}\right) \frac{1}{x^{4}}
$$

Next we need the kinetic energy of the $S O(8)$ field in the string theory. It can be obtained by compactifying the DiracBorn-Infeld action of the seven-branes on the $S^{3}$ around which they are wrapped. One finds

$$
\begin{aligned}
S & =-\mu_{7} \int d^{8} x e^{-\phi} \operatorname{tr} \sqrt{-\operatorname{det}\left(G_{a b}+2 \pi \alpha^{\prime} F_{a b}\right)} \\
& =\mu_{7}\left(2 \pi \alpha^{\prime}\right)^{2} \int d^{8} x e^{-\phi} \sqrt{-G} \frac{1}{4} \operatorname{tr} F^{2}+\cdots .
\end{aligned}
$$

Normalizing the $S O(8)$ generators as $\operatorname{tr}\left(T^{a} T^{b}\right)=\lambda \delta^{a b}$, rescaling the (induced) metric $G_{a b} \rightarrow \alpha^{\prime} \sqrt{8 \pi g_{s} N} G_{a b}$ and integrating over the unit $S^{3}$ of volume $2 \pi^{2}$ gives

$$
\begin{aligned}
S & =\lambda \frac{N}{16 \pi^{2}} \int d^{5} x \sqrt{-G} F_{\mu \nu}^{a} F^{a \mu \nu} \\
& \equiv \frac{1}{4 g_{S O(8)}^{2}} \int d^{5} x \sqrt{-G} F_{\mu \nu}^{a} F^{a \mu \nu} .
\end{aligned}
$$

From here we find the $S O(8)$ gauge coupling in the lowenergy five-dimensional effective theory

$$
g_{S O(8)}^{2}=\frac{1}{\lambda} \frac{4 \pi^{2}}{N} .
$$

We now compute the current-current correlation function using the AdS-CFT correspondence. We follow [13] and obtain

$$
\left\langle J_{\mu}^{a}(x) J_{\nu}^{b}(0)\right\rangle=\delta^{a b} \frac{1}{2 \pi^{2} g_{S O(8)}^{2}}\left(\eta_{\mu \nu} \square-\partial_{\mu} \partial_{\nu}\right) \frac{1}{x^{4}} .
$$

Comparison with the field theory result (4.2) gives

$$
\delta^{a b} \frac{8 \pi^{2}}{g_{S O(8)}^{2}}=2 N \operatorname{tr}\left(t^{a} t^{b}\right)
$$

which leads to $\operatorname{tr}\left(T^{a} T^{b}\right)=\operatorname{tr}\left(t^{a} t^{b}\right)$. This verifies that the relative normalizations are indeed the same.

After having shown the agreement of the relative normalization of the field theory and the string theory results, we will now turn to verify that the overall normalization also agrees. To this end we must properly normalize the $U(1)_{\mathcal{R}}$ gauge field in the low-energy five-dimensional action. The quadratic action for the massless gauge fields has been computed in [35], with the result 


$$
\begin{aligned}
S & =\frac{4(2 N)^{2}}{(2 \pi)^{5}} \int_{A d S_{5}} d^{5} x \sqrt{-g} \frac{\pi^{3}}{24} \frac{1}{3}\left(-\frac{1}{4} F(A)^{2}\right) \\
& \equiv-\frac{1}{4 g_{S G}^{2}} \int_{A d S_{5}} d^{5} x \sqrt{-g} F\left(A^{\mathcal{R}}\right)^{2} .
\end{aligned}
$$

$g_{S G}$ is defined to be the $U(1)$ gauge coupling constant of the $d=5$ low-energy theory, $A$ is the massless vector field defined above, and $A^{\mathcal{R}}$ is the rescaled gauge field which couples to $\mathcal{R}^{\mu}$. In the units used here, the overall prefactor comes from the term $1 / 2 \kappa^{2}$ in front of the $d=5$ supergravity action. To determine $g_{S G}$ we will follow once more the procedure of [13]. This means that we compute the two-point function $\left\langle\mathcal{R}^{\mu} \mathcal{R}^{\nu}\right\rangle$ in the four-dimensional field theory, which is proportional to the central charge in the $\mathcal{R}^{\mu}(x) \mathcal{R}^{\nu}(0)$ operator product. We find, to leading order in $1 / N$,

$$
\left\langle\mathcal{R}_{\mu}(x) \mathcal{R}_{\nu}(0)\right\rangle=-\frac{8 N^{2}}{(2 \pi)^{4}}\left(\square \eta_{\mu \nu}-\partial_{\mu} \partial_{\nu}\right) \frac{1}{x^{4}} .
$$

This can either be extracted from [37] or computed directly. This one-loop result is exact, due to the superconformal sym- metry. Comparison with the AdS computation (using again the results of [13]) gives $g_{S G}=\pi / N$. Using Eq. (4.8) and the definition of $\eta$ this leads to $\eta=1 / 2$. Plugging this into Eq. (3.13) we find exact agreement between Eqs. (2.8) and (3.13), verifying the AdS-CFT correspondence to this order.

\section{ACKNOWLEDGMENTS}

This work was supported in part by the US-Israel Binational Science Foundation, by GIF, the German-Israeli foundation for Scientific Research, by the Israel Science Foundation, and by the EEC under TMR contract ERBFMRXCT96-0045. The work of O.A. was supported in part by DOE grant DE-FG02-96ER40559. The work of J.P. was supported in part by Polish State Committee for Scientific Research (KBN) under contract 2P 03 B03 715 (1998-2000). J.P. would like to thank the Alexander-von-Humboldt Foundation for financial support and S.T. Tel Aviv University for hospitality during the initial stages of this work. We would like to thank S. Kuzenko, A. Schwimmer, C. Scrucca and in particular C.-S. Chu and J. Maldacena for useful discussions.
[1] J. M. Maldacena, Adv. Theor. Math. Phys. 2, 231 (1998).

[2] S. S. Gubser, I. R. Klebanov, and A. M. Polyakov, Phys. Lett. B 428, 105 (1998).

[3] E. Witten, Adv. Theor. Math. Phys. 2, 253 (1998).

[4] G. 't Hooft, Nucl. Phys. B72, 461 (1974).

[5] S. Coleman, Aspects of Symmetry (Cambridge University Press, Cambridge, England, 1985).

[6] O. Aharony and E. Witten, J. High Energy Phys. 11, 018 (1998)

[7] O. Aharony, M. Berkooz, D. Kutasov, and N. Seiberg, J. High. Energy. Phys. 10, 004 (1998).

[8] T. Banks and M. B. Green, J. High Energy Phys. 05, 002 (1998).

[9] M. Bianchi, M. B. Green, S. Kovacs, and G. Rossi, J. High Energy Phys. 08, 013 (1998).

[10] N. Dorey, V. V. Khoze, M. P. Mattis, and S. Vandoren, Phys. Lett. B 442, 145 (1998).

[11] N. Dorey, T. J. Hollowood, V. V. Khoze, M. P. Mattis, and S. Vandoren, "Multi-instantons and Maldacena's Conjecture," hep-th/9810243.

[12] D. Anselmi and A. Kehagias, "Subleading Corrections and Central Charges in the AdS/CFT Correspondence,', hep-th/9812092.

[13] D. Z. Freedman, S. D. Mathur, A. Matusis, and L. Rastelli, Nucl. Phys. B546, 96 (1999).

[14] M. Henningson and K. Skenderis, J. High Energy Phys. 07, 023 (1998).

[15] S. S. Gubser, Phys. Rev. D 59, 025006 (1999).

[16] E. Witten, J. High Energy Phys. 07, 006 (1998).

[17] S. Kachru and E. Silverstein, Phys. Rev. Lett. 80, 4855 (1998).

[18] A. Sen, Nucl. Phys. B475, 562 (1996).

[19] T. Banks, M. R. Douglas, and N. Seiberg, Phys. Lett. B 387, 278 (1996).

[20] O. Aharony, J. Sonnenschein, S. Theisen, and S. Yankielo- wicz, Nucl. Phys. B493, 177 (1997).

[21] M. R. Douglas, D. A. Lowe, and J. H. Schwarz, Phys. Lett. B 394, 297 (1997).

[22] A. Fayyazuddin and M. Spalinski, Nucl. Phys. B535, 219 (1998).

[23] O. Aharony, A. Fayyazuddin, and J. M. Maldacena, J. High Energy Phys. 07, 013 (1998).

[24] J. Polchinski, String Theory (Cambridge University Press, Cambridge, England, 1998), Vol. II.

[25] L. Alvarez-Gaumé and E. Witten, Nucl. Phys. B234, 269 (1984).

[26] M. Grisaru, in Recent Developments in Gravitation (Cargese Lectures, 1978), edited by M. Levy and S. Deser (Plenum Press, New York, 1979), p. 577, and references therein.

[27] D. Anselmi, D. Z. Freedman, M. T. Grisaru, and A. A. Johansen, Nucl. Phys. B526, 543 (1998).

[28] D. Anselmi, J. Erlich, D. Z. Freedman, and A. A. Johansen, Phys. Rev. D 57, 7570 (1998).

[29] D. Anselmi, “Quantum Closed Algebras and Closed Conformal Field Theory,', hep-th/9811149.

[30] M. B. Green, J. A. Harvey, and G. Moore, Class. Quantum Grav. 14, 47 (1997).

[31] J. Morales, C. Scrucca, and M. Serone, "Anomalous Couplings for $D$-branes and $O$-planes,' hep-th/9812071.

[32] B. Stefanski, "Gravitational couplings of $D$-branes and O-planes,'” hep-th/9812088.

[33] C. Vafa, Nucl. Phys. B469, 403 (1996).

[34] C. Bachas, 'Lectures on D-branes,'” hep-th/9806199.

[35] G. Arutyunov and S. Frolov, "Quadratic Action for type IIB Supergravity on $A d S_{5} \times S^{5}$," hep-th/9811106.

[36] H. Kim, L. Romans, and P. van Nieuwenhuizen, Phys. Rev. D 32, 389 (1985).

[37] D. Anselmi, D. Z. Freedman, M. T. Grisaru, and A. A. Johansen, Phys. Lett. B 394, 329 (1997). 